\title{
RHINOLOGY
}

\section{Olfactory dysfunction in primary Sjogren's syndrome and its correlation with dry eye}

\author{
Difunzione olfattiva nella sindrome di Sjogren e correlazione con la xeroftalmia \\ Yunus E. Topan¹, Banu Bozkurt², Sema Yılmaz³, Çağdaş Elsürer¹, Sona Gorcuyeva², Mete K. Bozkurt¹ \\ ${ }^{1}$ Selcuk University School of Medicine, Otolaryngology Dept., Konya, Turkey; ${ }^{2}$ Selcuk University School of Medicine, Ophthalmology \\ Dept., Konya, Turkey; ${ }^{3}$ Selcuk University School of Medicine, Rheumatology Dept., Konya, Turkey
}

\section{SUMMARY}

Objective. To evaluate the olfactory function in primary Sjögren's syndrome (pSS) patients and investigate its correlation with dry eye parameters.

Methods. Thirty-eight pSS patients $(49.47 \pm 10.06$ years $)$ and 20 healthy volunteers $(47.40 \pm 8.92$ years $)$ were enrolled in the study. All participants underwent ENT and eye examinations including a modified Connecticut Chemosensory Clinical Research Center (CCCRC) test, tear break-up time (TBUT), ocular surface staining (OSS) and Schirmer test. The parameters were compared between the two groups using Student-t test, and Pearson test was used to evaluate the correlations.

Results. Mean Schirmer and TBUT values were $2.39 \pm 1.48 \mathrm{~mm} / 5 \mathrm{~min}$ and $3.66 \pm 1.5 \mathrm{sec}$ in $\mathrm{pSS}$ and $18.30 \pm 6.16 \mathrm{~mm} / 5 \mathrm{~min}$ and $14.60 \pm 3.64 \mathrm{sec}$ in healthy subjects $(\mathrm{p}<0.001$, both). There was a significant decrease in mean odour threshold, odour identification, CCCRC and VAS scores in the pSS group $(\mathrm{p}<0.001)$. Dry eye parameters showed moderate correlations with CCCRC parameters $(\mathrm{r}=0.4-0.6, \mathrm{p}<0.001)$ and olfaction VAS score $(\mathrm{r}=0.4-0.75, \mathrm{p}<0.05)$.

Conclusions. There is a mild clinical impairment in smell sense in patients with pSS which seems to be correlated with dry eye parameters. Therefore, smell complaints should be queried in pSS patients suffering from severe dry eye.

KEY WORDS: primary Sjögren's syndrome, dry eye, olfactory dysfunction, CCCRC

\section{RIASSUNTO}

Obiettivo. Valutare la funzione olfattiva nei pazienti con sindrome di Sjögren primaria (pSS) e indagare la sua correlazione con la xeroftalmia.

Metodi. Nello studio sono stati arruolati trentotto pazienti con pSS $(49,47 \pm 10,06$ anni) e 20 volontari sani $(47,40 \pm 8,92$ anni). Tutti i partecipanti sono stati sottoposti a vista ORL e oculistica, inclusiva del test del Connecticut Chemosensory Clinical Research Center (CCCRC), Tear Break-up Time (TBUT), colorazione della superficie oculare (OSS) e test di Schirmer. I parametri sono stati confrontati tra i due gruppi utilizzando il test $t$ di Student e il test di Pearson è stato utilizzato per valutare le correlazioni.

Risultati. I valori medi di Schirmer e TBUT erano 2,39 $\pm 1,48 \mathrm{~mm} / 5 \mathrm{~min}$ e 3,66 $\pm 1,5 \mathrm{sec}$ nel gruppo pSS e 18,30 \pm 6,16 mm/5 min e 14,60 \pm 3,64 sec nei soggetti sani $(p<0,001$, entrambi). C'è stata una diminuzione statisticamente significativa della soglia media dell'odore, dell'identificazione dell'odore, dei punteggi CCCRC e VAS nel gruppo pSS $(p<0,001)$. I parametri dell'occhio secco hanno mostrato correlazioni moderate con $i$ parametri CCCRC $(r=0,4-0,6, p<0,001) e$ il punteggio VAS dell'olfatto $(r=0,4-0,75$, $p<0,05)$.

Conclusioni. Esiste una lieve compromissione clinica del senso dell'olfatto nei pazienti con PSS che sembra essere correlata alla xeroftalmia. Pertanto, i disturbi dell'olfatto dovrebbero essere valutati nei pazienti con PSS che soffrono di grave secchezza oculare.

PAROLE CHIAVE: sindrome di Sjögren primaria, xeroftalmia, disfunzione olfattiva, CCCRC
Received: December 29, 2020

Accepted: June 4, 2021

Correspondence

Mete Kaan Bozkurt

Selcuk University, School of Medicine, Department of Otolaryngology Head \& Neck Surgery, Konya, Turkey

Tel. +90 3322243947

E-mail: metekaanbozkurt@gmail.com

Funding

None.

Conflict of interest

The Authors declare no conflict of interest.

How to cite this article: Topan YE, Bozkurt B, Yılmaz S, et al. Olfactory dysfunction in primary Sjogren's syndrome and its correlation with dry eye. Acta Otorhinolaryngol Ital 2021;41:443-449. https://doi.org/10.14639/0392-100X-N1344

(C) Società Italiana di Otorinolaringoiatria e Chirurgia Cervico-Facciale

\section{c) (i)}

This is an open access article distributed in accordance with the CC-BY-NC-ND (Creative Commons Attribution-NonCommercial-NoDerivatives 4.0 International) license. The article can be used by giving appropriate credit and mentioning the license, but only for non-commercial purposes and only in the original version. For further information: https:// creativecommons.org/licenses/by-nc-nd/4.0/deed.en 


\section{Introduction}

Primary Sjogren's syndrome (pSS) is a chronic, autoimmune, systemic disease characterised by rhinitis sicca, symptoms of dry eyes and dry mouth driven by lymphocytes and autoantibodies targeting the exocrine glands, primarily the lacrimal and salivary glands ${ }^{1-5}$. The revised international classification criteria for diagnosis of SS are typical ocular and oral symptoms, ocular signs (Schirmer's test and/or ocular staining score), reduced saliva secretion, histopathology of minor salivary glands showing lymphocytic infiltration and the presence of autoantibodies against Ro/SS-A and La/ SS-B ${ }^{6}$. The disease is named as primary SS (pSS) in the absence of accompanying autoimmune connective tissue disease, such as rheumatoid arthritis (RA), systemic lupus erythematosus (SLE) and scleroderma. The prevalence of pSS is estimated to be $0.06 \%{ }^{7}$. The disorder mainly affects women, with a female to male ratio as nine. As a result of permenant destruction of lacrimal gland acini and ducts, tear production is reduced and patients complain of dryness, discomfort and foreign body sensation in the eye, visual fluctuation with blinking, blurred vision and impaired quality of life (QOL) ${ }^{5,8}$. In addition, SS can lead to corneal ulcers, scleritis, uveitis, retinal vasculitis and optic neuritis.

Senses of smell and taste are very important for nutrition, in terms of recognition of foods, being one of the first warning systems against chemicals, avoiding detrimental effects of these substances and involuntary ingestion and early digestion of dietary substances. Smell sensory processing system can detect and discriminate thousands of low molecular mass compounds (odourants). Via biochemical and electrophysiological processes, the molecular information converts into smell sensation ${ }^{9}$.

Olfaction affects eating behaviour, digestive system physiology, gastric secretion and nervous system ${ }^{9}$. Olfactory dysfunction can manifest as reduced ability, disturbance or absence of smell. Olfaction can be impaired by transmissional pathologies of nasal and paranasal sinuses, sensorineural pathologies influencing the olfactory epithelium and bulbus, aging, chronic smoking, alcohol abuse, metabolic and autoimmune diseases and olfactory dysfunction has a negative effect on QOL ${ }^{10,11}$.

Saliva and nasal mucus are important in maintaining normal taste and smell acuity through their effects on taste buds imbedded in the oral epithelium and olfactory cells found in the nasal cavity. In SS, progressive involvement of exocrine glands with loss of secretion might impair the special senses of smell and taste. Patients with SS frequently report dry mouth, taste impairment, nasal dryness, odour change, and hyposmia ${ }^{12-16}$. Low production of nasal mucous might lead to nasal obstruction, increased nasal resist- ance and a decrease in olfactory sensation, via preventing the transport of olfactory molecules to the olfactory epithelium. In this study, we evaluated the olfactory function in pSS patients using both a subjective Visual Analog Scale (VAS) and a modified Connecticut Chemosensory Clinical Research Center (CCCRC) test and compared these findings with those age- and gender-matched healthy controls. We further investigated the correlation of smell dysfunction with dry eye parameters.

\section{Materials and methods}

The study was approved by the Selcuk University Research Ethics Committee (2018/20) and followed the tenets of 1964 Helsinki Declaration. A total of 38 patients diagnosed with pSS in the Rheumatology Department of Selcuk University Medical Faculty, all fulfilling the American-European Consensus classification criteria ${ }^{6}$ and age- and sexmatched 20 healthy volunteers were included in the study. Written informed consent was obtained from all participants. Patients were using systemic immunosupressive/immunoregulatory treatment including steroids, hydroxycloroquine and methothrexate. Exclusion criteria were history of head trauma or stroke, chemotherapy or radiotherapy in head and neck region, hepatitis $\mathrm{C}$ infection, AIDS, preexisting lymphoma, sarcoidosis, graft versus host disease, nasal polyposis, advanced septal deviation, active upper respiratory tract infection, allergic rhinitis, use of anticholinergic drugs, previous nasal surgery, current smoking and chronic alcohol use. Patients with secondary SS were not included in the study.

\section{ENT examination}

Both sides of nasal cavities were evaluated endoscopically using a Storz $0^{\circ}$ endoscope. AR was performed with RhinoMetrics ${ }^{\circledR}$ SRE2000 (Lynge, Denmark). The nasal volume from 0 to $2.2 \mathrm{~cm}$ (VOL1), from $2.2 \mathrm{~cm}$ to $5.4 \mathrm{~cm}$ (VOL2), total nasal volume from 0 to $5.4 \mathrm{~cm}$ (TVOL), the minimum cross-sectional area of the anterior (MCA1) and the minimum cross-sectional area of the posterior (MCA2) were measured from each nasal cavity within the distance of 2.2 and $5.4 \mathrm{~cm}$ from the nostrils. The test was repeated 3 times and mean values were recorded. Subjects were asked to score their smell perception on VAS, where self-reported smell was scored as 0 indicating no smell perception up to 10 showing very good smell perception. Objective olfactory function was evaluated by a modified CCCRC test which includes a butanol threshold test and odour identification test using common odours ${ }^{17}$, which was validated in Turkish population by Veyseller et al. ${ }^{18}$. Threshold testing was performed with solutions of 1-butanol in deionised 
water, decreasing in 8 steps. The test result was expressed as threshold score from 0 to 8 . In the identification test, 8 well-known odourants which contains peanut butter, soap, mothballs, Vicks, chocolate, coffee, cinnamon and baby powder were presented within $180 \mathrm{ml}$ opaque jars to the intact trigeminal nerve function test person in the same manner. As the ability to sense Vicks VapoRub indicates intact trigeminal nerve function and was easily identified by all subjects, it was not included in the final score ${ }^{18}$. Possible scores ranged from 0 to 7 items correctly identified. Scores for both nostrils were averaged to arrive at the final score. Butanol threshold and identification tests were averaged to obtain a CCCRC score.

\section{Eye examination}

All subjects underwent a detailed ophthalmological examination, including Schirmer test, tear break up time (TBUT) test and ocular surface staining (OSS). Schirmer test is a standard measure of aqueous tear production and is safe, easy to perform and inexpensive. Whatman's filter paper (No. 41) of $5 \times 35 \mathrm{~mm}$ length was placed in the outer $1 / 3$ portion of both lower eyelid fornices and the length of wetting was measured and $\leq 5 \mathrm{~mm}$ at the end of 5 minutes was defined as definitely decreased tear production. TBUT is an indicator of tear film stability and commonly used in the assessment of dry eye disease. Sodium fluorescein is installed into the lower conjunctiva and the patient is asked to look straight ahead and blink a few times. The tear film is observed under blue light and the time between the last blink and the first dry spot is recorded. More than $10 \mathrm{sec}-$ onds is considered normal, 5 to 10 seconds marginal and $<5$ seconds was considered as abnormal TBUT. According to the Oxford Scale, the OSS was graded between stage 0 and 5 and then categorised as mild (stage 0-1), moderate (stage 2 or 3), or severe (stage 4-5).

\section{Statistical analysis}

Statistical analysis was performed using SPSS 23.0 (SPSS/ IBM, Inc., Chicago, IL, USA) for Microsoft Windows. Data are expressed as mean \pm standard deviation, range (min-max) and percentage. The parameters were compared between pSS and healthy using Student-t test. Since the data were normally distributed, Pearson's test was used to evaluate the correlation between smell test parameters and dry eye tests and a $p$ value $<0.05$ was considered as statistically significant.

\section{Results}

The pSS group included 35 females $(92.11 \%)$ and 3 males $(7.89 \%)$ with a mean age of $49.47 \pm 10.06$ years (range 26-73 years), and the control group included 19 females (95\%) and 1 male (5\%) with a mean age of $47.40 \pm 8.92$ years (range 35-68 years). There were no differences between the two groups in terms of age and gender $(p=0.44$ and 0.57 , respectively). The mean duration of SS diagnosis and treatment was found as 54.03 months (12 months-240 months). The systemic medications were hydroxychloroquine, methotrexate, sulfasalazine, azathioprine and steroids. Nine subjects in the pSS group (23.7\%) and 4 subjects in the control group (20\%) were former smokers $(\mathrm{p}=0.75)$. None of the healthy subjects had complaints of dry eye, dry mouth, dysphagia, nasal dryness, taste or smell loss. Mean Schirmer and TBUT values were significantly lower in $\mathrm{pSS}$ group $(2.39 \pm 1.48 \mathrm{~mm} / 5 \mathrm{~min}$ and $3.66 \pm 1.5 \mathrm{sec}$, respectively) compared to healthy subjects $(18.30 \pm 6.16 \mathrm{~mm} / 5$ min and $14.60 \pm 3.64 \mathrm{sec}$, respectively) $(\mathrm{p}<0.001$, both $)$ (Tab. I). According to the Oxford scale, the OSS was mild in $15 \mathrm{pSS}$ subjects $(39.5 \%)$, moderate in $14(36.8 \%)$ and severe in 9 subjects $(23.7 \%)$.

The pSS subjects had a lower mean self-reported smell score on VAS than the control group $(7.45 \pm 1.01$ and $9.35 \pm 0.59$, respectively) $(\mathrm{p}<0.05)$. Mean odour threshold, odour identification and CCCRC scores were significantly lower in the pSS group $(7.05 \pm 0.73,5.32 \pm 0.99$ and $6.05 \pm 0.63$, respectively) than the control group $(7.95 \pm 0.22,6.45 \pm 0.60$ and $6.73 \pm 0.30$, respectively) $(\mathrm{p}<0.001)$ (Tab. II). The post hoc power was $99 \%$ (1-b > 0.99), which indicates that our results were highly reliable.

Mean VAS score, odour threshold, odour identification and CCCRC scores were found to be significantly lower in pSS patients with severe OSS $(6.78 \pm 0.83,6.67 \pm 0.87$, $4.78 \pm 0.97$ and $5.67 \pm 0.75$, respectively) compared to mild OSS $(7.73 \pm 0.7,7.33 \pm 0.62,5.87 \pm 0.64$ and $6.40 \pm 0.34$, respectively) $(\mathrm{p}<0.05$, all), while only odour identification and CCCRC scores differed between mild and moder-

Table I. Demographic data and dry eye test results.

\begin{tabular}{lccc} 
& pSS group $\mathbf{n}=\mathbf{3 8}$ & Controls $\mathbf{n}=\mathbf{2 0}$ & p value \\
Age (years) (min-max) & $49.47 \pm 10.06(26-73)$ & $47.40 \pm 8.92(35-68)$ & 0.44 \\
Gender (female n/\%) & $35(92.11 \%)$ & $19(95 \%)$ & 0.57 \\
Schirmer test (mm/5min) & $2.39 \pm 1.48(1-7)$ & $18.30 \pm 6.16(10-30)$ & $<0.001$ \\
TBUT (sec) & $3.66 \pm 1.5(2-8)$ & $14.60 \pm 3.64(10-21)$ & $<0.001$ \\
\hline
\end{tabular}


Table II. Olfactory test and acustic rhinometry results of pSS and control group (Mean \pm SD).

\begin{tabular}{lccc} 
& pSS group & Control group & P value \\
Odour threshold score & $7.05 \pm 0.73(5-8)$ & $7.95 \pm 0.22(7-8)$ & $<0.001$ \\
Odour identification score & $5.32 \pm 0.99(3-7)$ & $6.45 \pm 0.60(5-7)$ & $<0.001$ \\
CCCRC value & $6.05 \pm 0.63(4-7)$ & $6.73 \pm 0.30(6-7)$ & $<.001$ \\
Odour detection VAS score & $7.45 \pm 1.01(5-10)$ & $9.35 \pm 0.59(8-10)$ & 0.001 \\
MCA1 & $0.36 \pm 0.17(0.04-0.74)$ & $0.40 \pm 0.13(0.16-0.74)$ & 0.004 \\
MCA2 & $0.42 \pm 0.25(0.04-1.44)$ & $0.65 \pm 0.31(0.15-1.52)$ & 0.14 \\
Right Vol1 & $1.62 \pm 0.58(0.52-3.51)$ & $1.83 \pm 0.38(1.28-2.52)$ & 0.001 \\
Right Vol2 & $5.11 \pm 3.33(0.51-16.42)$ & $9.01 \pm 5.13(3.44-24.97)$ & 0.001 \\
Right total volume & $6.73 \pm 3.74(1.03-18.46)$ & $1.81 \pm 5.28(5.13-26.89)$ & 0.19 \\
Left Vol1 & $1.81 \pm 0.9(0.77-3.65)$ & $7.34 \pm 3.26(2.30-14.44)$ & $0.49(0.92-3.03)$ \\
Left Vol2 & $5.94 \pm 2.53(2.75-13.12)$ & $8.94 \pm 3.6(3.80-16.17)$ \\
Left total volume & $7.75 \pm 2.89(3.52-16.77)$ & 0.18 \\
\hline
\end{tabular}

ate OSS groups $(\mathrm{p}<0.05)$. Schirmer test, TBUT and Oxford OSS score showed significant correlations with odour threshold $(\mathrm{r}=0.49,0.55$ and $0.40, \mathrm{p}<0.05$ respectively), odour identification score $(\mathrm{r}=0.6,0.61$ and $0.51, \mathrm{p}<0.001$, respectively), CCCRC score $(\mathrm{r}=0.56,0.58$ and 0.54 , $\mathrm{p}<0.001$, respectively) and olfaction VAS score $(\mathrm{r}=0.75$, 0.75 and $0.43, \mathrm{p}<0.05$ ). Disease duration showed negative correlation with odour threshold $(\mathrm{r}=-0.53, \mathrm{p}<0.001)$, odour identification score $(\mathrm{r}=-0.48, \mathrm{p}<0.001)$, CCCRC score $(\mathrm{r}=-0.55, \mathrm{p}<0.001)$ and olfaction VAS score $(\mathrm{r}=-0.49, \mathrm{p}<0.001)$.

Among AR parameters, mean MCA2, right VOL2 and right total volume were significantly lower in the pSS group compared to the control group $(\mathrm{p}<0.05)$. No correlations were found between AR parameters and CCCRC scores $(p>0.05)$, while a mild correlation was found between olfaction VAS score and MCA2 $(r=0.32, p=0.014)$, right $\operatorname{VOL} 2(\mathrm{r}=0.28, \mathrm{p}=0.031)$ and right total volume $(\mathrm{r}=0.27$, $\mathrm{p}=0.039)$.

\section{Discussion}

Sjogren's syndrome has a negative impact on smell and taste function ${ }^{12-16}$. Henkin et al. ${ }^{12}$ studied taste and smell thresholds and forced scaling levels in 29 patients with SS and and in 10 patients with various diseases of the parotid glands. They showed significant taste and smell loss in patients with xerostomia and rhinitis sicca, and treatment with cyclophosphamide or X-ray were shown to improve taste or smell function if the clinical signs and symptoms of xerostomia or rhinitis sicca concomitantly improved. Weiffenbach et al. ${ }^{13}$ used smell identification test in $30 \mathrm{pSS}$ patients and 60 healthy subjects and found decreased olfaction in $26.7 \%$ of pSS subjects, while only $6.6 \%$ of the controls showed impairment in smell function. Kamel et al. ${ }^{14}$ assessed smell and taste perception using University of Pennsylvania Smell Threshold test and impregnated taste strips of 4 tastes (sweet, sour, salty and bitter) in 28 pSS patients and 37 healthy subjects. They showed one point decrease in odour detection threshold and 3.5 point decrease in taste threshold in SS group compared to control group. Forty-three percent of SS patients were hyposmic $(<4.5)$ compared with $19 \%$ of the healthy group $(\mathrm{p}=0.036)$. The authors concluded that clinically impairment of chemosensory perception occurred in SS subjects, which contributed to a reduced health related QOL evaluated by the Short Form $12{ }^{14}$. Decreased mucin (odourant carrier), recurrent rhinosinusitis and immunological mechanisms were proposed to explain decreased smell and taste disorder in SS subjects. In the study of Rusthen et al. ${ }^{15}$, mean odour VAS score and odour identification were 6.7 and 8.8 in pSS patients and 8.3 and 10.7 in healthy subjects $(\mathrm{p}<0.05)$ and $12.9 \%$ of the patients were classified as anosmic. In the meta-analysis of Al-Ezzi et al. ${ }^{16}$ based on 5 studies with 378 participants, standardised mean differences (SMD) between pSS and healthy controls were -0.78 (95\% CI -1.29 to -0.27 ) for smell; -1.01 (95\% CI -1.54 to -0.49$)$ for taste; -0.93 (95\% CI -1.22 to -0.64 ) for total sexual function; -1.28 (95\% CI -1.65 to -0.90$)$ and -0.83 (95\% CI -1.27 to -0.40 ) for physical and mental component of the QOL respectively; and $0.61(95 \%$ CI $0.02,1.20)$ and 0.79 (95\% CI 0.43 to 1.15 ) for anxiety and depression, respectively. The authors concluded that pSS deteriorates smell, taste, sexual function and QOL in women. Singh et al. ${ }^{19}$ investigated olfactory and gustatory function, salivary secretion rates, burning mouth sensation and halitosis in 58 pSS patients, 22 non-Sjogren's syndrome sicca patients and 57 age-matched healthy controls. Olfactory and gustatory 
functions were assessed using self-reported perception of sense of smell and taste on VAS, 12-stick identification test and taste strips. Patients with pSS had a mild impairment in smell sense compared to the control group (median 10/12 and $11 / 12$, respectively, $\mathrm{p}=0.007$ ) and median gustatory and VAS scores were lower in patients with pSS (median 20 and 7, respectively) than the healthy subjects (median 26 and 8 , respectively) $(\mathrm{p}<0.05)$. A recent study by Šijan Gobeljić et al. ${ }^{20}$ showed that patients with pSS had significantly lower self-reported VAS smell score $(8.6 \pm 2.2)$ and VAS taste score $(8.5 \pm 2.1)$ than healthy controls $(9.6 \pm 0.7$, $\mathrm{p}=0.016$ and $9.5 \pm 0.7, \mathrm{p}=0.014$, respectively). Using Sniffin Sticks test and taste strips, 3.8\% of patients were shown to have anosmia with scores between $0-5,36.5 \%$ had hyposmia with scores between 6-9, 34.0\% had ageusia for sweetness, $10.6 \%$ for sourness, $10.0 \%$ for saltiness and $19.1 \%$ for bitterness. Several factors may contribute to the olfactory dysfunction in the SS patients, such as nasal dryness, decreased mucin (an odourant carrier) and recurrent rhinosinusitis ${ }^{14}$. In case of decreased nasal secretion, olfactory molecules can not dissolve within mucus and their transport to the olfactory epithelium is disturbed. Secretory immunoglobulins, distributed on the mucous film lining the respiratory epithelium, neutralise viruses and bacterial surface antigens, favouring their phagocytosis. Decreased nasal mucous leads to a disruption in defense barrier and causes recurrent airway infections. Nasal dryness might also lead to epistaxis, crust formation and nasal blockage, all of which contribute to impaired olfactory function.

However, there are also contradictory studies, which did not reveal olfactory dysfunction in subjects with SS. In the study of Rasmussen et al. ${ }^{21}$, although $39 \%$ of pSS patients complained of dryness in the nose and $44 \%$ of nasal crust formation, no significant difference was found between pSS and healthy subjects in odour detection using coffee or mucociliary clearance. In the study of Midilli et al. ${ }^{22}$, which investigated nasal and paranasal findings in $77 \mathrm{pSS}$ patients and 77 control subjects, no significant differences were found in nasal clearance analysis with saccharin test and 5 component smell discrimination test between patients with SS and healthy subjects. In the studies of Rasmussen et al. ${ }^{21}$ and Midilli et al. ${ }^{22}$, olfactory dysfunction was evaluated with more simpler smell tests and no information was given about the severity of disease in pSS participants. In a recent study by Eren et al. ${ }^{23}$, nasal dryness, postnasal drip, and a decreased smell sense were remarkable in the SS group $(\mathrm{p}<0.05)$. Intranasal Schirmer test scores were lower in patients with SS $(8.4$ and $8 \mathrm{~mm}$, in right and left nasal cavities) compared to healthy controls $(11.7 \mathrm{~mm}$, both nasal cavities) $(\mathrm{p}=0.041)$; however CCCRC scores did not differ between the 2 groups. Intranasal Schirmer wetting distances of 6 to $18 \mathrm{~mm}$ are considered normal, which means most of the the SS subjects included in the study of Eren et al. ${ }^{23}$ might not have severe dryness in the nasal mucosa. They found a trend toward a positive correlation between olfactory function and intranasal Schirmer test scores, but it was not statistically significant.

In our study, olfactory function was evaluated in 38 patients diagnosed with pSS and the results were compared with age and sex-matched 20 healthy subjects. Mean subjective odour detection VAS score was 7.45 in pSS and 9.35 in the control group $(\mathrm{p}<0.05)$. Using CCCRC test, mean odour threshold, odour identification and CCCRC scores were significantly lower in the pSS group $(\mathrm{p}<0.001)$. However, the difference between the groups is around one point in all parameters which in fact may not be noticed by the patient. Most of the AR parameters did not differ between pSS and the control group and no correlations were found between AR parameters and smell tests ( $p>0.05$ ), which supports the hypothesis that olfactory dysfunction in pSS is not due to obstructive causes.

Mean CCCRC parameters in our control group was higher than normative values of 426 Turkish healthy volunteers ${ }^{18}$, which accepted maximum threshold scoring as 7, while it was 8 in our study. In addition, $37.1 \%$ of those subjects were smokers, while none of our subjects were current smokers.

In the literature, there is no study investigating the relationship between dry eye and olfactory function tests. In our study, the decrease in mean VAS score, odour threshold, odour identification and CCCRC scores were more remarkable in pSS patients with severe OSS $(6.78 \pm 0.83$, $6.67 \pm 0.87,4.78 \pm 0.97$ and $5.67 \pm 0.75$, respectively) compared to mild OSS $(7.73 \pm 0.7,7.33 \pm 0.62,5.87 \pm 0.64$ and $6.40 \pm 0.34$, respectively) ( $<<0.05$, all). The Schirmer test, TBUT and OSS score showed significant correlations with CCCRC test parameters and olfaction VAS score, which shows that smell loss was more remarkable in SS patients suffering from severe dry eye disease. In clinical practice, a good correlation was found between ocular and oral involvement in patients with pSS. In pSS patients, Tashbayev et al. ${ }^{24}$, showed that subjective oral dryness significantly correlated with ocular dryness and stimulated saliva secretion rate was significantly correlated with Schirmer I $(\mathrm{r}=0.419)$. Ocular dryness might also be associated with nasal dryness, which might be the cause of smell loss in SS patients. One of our limitations was the relatively small number of patients in the study; however, the post-hoc power was greater than $80 \%(1-b>0.99)$, which indicates that the results were highly reliable. The lack of measurements of salivary secretion rate, mucociliary function and nasal Schirmer test were other limitations, which will be 
evaluated in a further study. Rheumatologists always refer pSS patients to ophthalmologists for evaluation of dry eye severity and ocular side effects of medications used in SS, mainly the adverse effects of hydroxychloroquine. Dry eye tests, especially Schirmer test, is routine in these patients, while ENT referral, nasal and ocular dryness tests are not always performed, generally done as part of an investigation. Therefore, dry eye is an indicator of pSS severity and deterioration in dry eye test parameters might be a marker for nasal involvement and smell impairment.

There are also complex interactions and some similarities between autoimmunity and the olfactory system. Patients with SLE, Sjogren's syndrome, polydermatomyositis and hereditary angioedema were shown to have disturbances in olfactory functions ${ }^{25}$. Genetics, hormonal and environmental factors may play a role in this relationship. Olfactory receptor gene clusters are located in proximity to MHCcomplex, a key locus for autoimmune disorders, suggesting both physical and functional linkage between smell loss and autoimmunity ${ }^{25,26}$.

In the study of Shoenfeld et al. ${ }^{27}$, using the Sniffin' Sticks test, patients with SLE were found to have a significant decrease in smell abilities compared with controls as $46 \%$ vs $25 \%$ were hyposmic and $10 \%$ vs none were anosmic, respectively. Magnetic resonance imaging (MRI) studies have shown involvement of the limbic system and functional areas of olfactory system, including amygdala and hippocampus might support this clinical observation. Immune-mediated mechanisms and drugs might be responsible for olfactory impairment. Bombini et al. ${ }^{28}$ using the Sniffin' Sticks test found olfactory dysfunction in $54.5 \%$ of 143 SLE, $59.3 \%$ of 57 systemic sclerosis (SSc) and $14.45 \%$ of 166 healthy volunteers $(\mathrm{p}<0.001)$ at study entry. Olfactory dysfunction was found to be associated with age, disease activity, inflammation and smaller hippocampi and amygdalae volumes using volumetric MRI and FreeSurfer ${ }^{\circledR}$ software. Mikulicz's disease is an immunoglobulin IgG4 systemic disease. In the study by Takano et al. ${ }^{29}$, of 44 patients, $45 \%$ had olfactory dysfunction even though they had no obstructive and inflammatory disease in nasal cavities and sinuses. In subjects with olfactory dysfunction, there were IgG4 positive plasmacytes in the nasal mucosa, which might support a linkage between smell loss and autoimmunity.

\section{Conclusions}

Our study showed that patients with pSS have a mild impairment in smell. Herein, we evaluated for the first time the relationship between dry eye tests and olfactory function parameters and showed that they were moderately cor- related. Olfactory dysfunction was more remarkable in pSS patients with moderate-severe ocular staining. Therefore, smell complaints must be queried in SS patients suffering dry eye.

\section{Acknowledgements}

We thank Asistant Prof. Umut Ece Arslan, Hacettepe University, Public Health Institute, Ankara, for her assistance in statistical analysis.

\section{References}

1 Kabasakal Y, Kitapçı̆̆lu G, Karabulut G, et al. Criteria sets for primary Sjogren's syndrome are not adequate for those presenting with extraglandular organ involvements as their dominant clinical features. Rheumatol Int 2017;37:675-684. https://doi.org/10.1007/ s00296-017-3691-8

2 Patel R, Shahane A. The epidemiology of Sjögren's syndrome. Clin Epidemiol 2014;6:247-255. https://doi.org/10.2147/CLEP.S47399

3 Vivino FB. Sjogren's syndrome: clinical aspects. Clin Immunol 2017;182:48-54. https://doi.org/10.1016/j.clim.2017.04.005

4 Fox PC. Autoimmune diseases and Sjogren's syndrome: an autoimmune exocrinopathy. Ann N Y Acad Sci 2007;1098:15-21. https://doi. org/10.1196/annals.1384.003

5 Bjordal O, Norheim KB, Rødahl E, et al. Primary Sjögren's syndrome and the eye. Surv Ophthalmol 2020;65:119-132. https://doi. org/10.1016/j.survophthal.2019.10.004

6 Vitali C, Bombardieri S, Jonsson R, et al. Classification criteria for Sjögren's syndrome: a revised version of the European criteria proposed by the American-European Consensus Group. Ann Rheum Dis 2002;61:554-558. https://doi.org/10.1136/ard.61.6.554

7 Qin B, Wang J, Yang Z, et al. Epidemiology of primary Sjögren's syndrome: a systematic review and meta-analysis. Ann Rheum Dis 2015;74:1983-1989. https://doi.org/10.1136/ annrheumdis-2014-205375

8 Akpek EK, Bunya VY, Saldanha IJ. Sjögren's syndrome: more than just dry eye. Cornea 2019;38:658-661. https://doi.org/10.1097/ ICO.0000000000001865

9 Hasin-Brumshtein Y, Lancet D, Olender T. Human olfaction: from genomic variation to phenotypic diversity. Trends Genet 2009;25:178184. https://doi.org/10.1016/j.tig.2009.02.002

10 Wrobel BB, Leopold DA. Clinical assessment of patients with smell and taste disorders. Otolaryngol Clin North Am 2004;37:1127-1142. https://doi.org/10.1016/j.otc.2004.06.010

11 Doty RL, Bromley SM. Effects of drugs on olfaction and taste. Otolaryngol Clin North Am 2004;37:1229-1254. https://doi.org/10.1016/j. otc.2004.05.002

12 Henkin RI, Talal N, Larson AL, et al. Abnormalities of taste and smell in Sjogren's syndrome. Ann Intern Med 1972;76:375-383. https://doi. org/10.7326/0003-4819-76-3-375

13 Weiffenbach JM, Fox PC. Odor identification ability among patients with Sjögren's syndrome. Arthritis Rheum 1993;36:1752-1754. https://doi.org/10.1002/art.1780361218

14 Kamel UF, Maddison P, Whitaker R. Impact of primary Sjogren's syndrome on smell and taste: effect on quality of life. Rheumatology (Oxford) 2009;48:1512-1514. https://doi.org/10.1093/rheumatology/ kep249 
15 Rusthen S, Young A, Herlofson BB, et al. Oral disorders, saliva secretion, and oral health-related quality of life in patients with primary Sjögren's syndrome. Eur J Oral Sci 2017;125:265-271. https://doi. org/10.1111/eos. 12358

16 Al-Ezzi MY, Pathak N, Tappuni AR, et al. Primary Sjögren's syndrome impact on smell, taste, sexuality and quality of life in female patients: a systematic review and meta-analysis. Mod Rheumatol 2017;27:623-629. https://doi.org/10.1080/14397595.2016.1249538

17 Cain WS, Gent JF, Goodspeed RB, et al. Evaluation of olfactory dysfunction in the Connecticut Chemosensory Clinical Research Center. Laryngoscope 1988;98:83-88. https://doi. org/10.1288/00005537-198801000-00017

18 Veyseller B, Ozucer B, Karaaltin AB, et al. Connecticut (CCCRC) olfactory test: normative values in 426 healthy volunteers. Indian J Otolaryngol Head Neck Surg 2014;66:31-34. https://doi.org/10.1007/ s12070-013-0632-Z

19 Singh PB, Young A, Homayouni A, et al. Distorted taste and impaired oral health in patients with sicca complaints. Nutrients 2019;11:264. https://doi.org/10.3390/nu11020264

20 Šijan Gobeljić M, Milić V, Pejnović N, et al. Chemosensory dysfunction, oral disorders and oral health-related quality of life in patients with primary Sjögren's syndrome: comparative cross-sectional study. BMC Oral Health 2020;20:187. https://doi.org/10.1186/ s12903-020-01169-5

21 Rasmussen N, Brofeldt S, Manthorpe R. Smell and nasal findings in patients with primary Sjögren's syndrome. Scand J Rheumatol Suppl 1986;61:142-145.
22 Midilli R, Gode S, Oder G, et al. Nasal and paranasal involvement in primary Sjogren`s syndrome. Rhinology 2013;51:265-267. https:// doi.org/10.4193/Rhino13.001

23 Eren E, Balcı K, Gerçik Ö, et al. Does nasal secretion decrease in Sjögren syndrome and does this affect nasal function? Laryngoscope 2021;131:370-373. https://doi.org/10.1002/lary.29022

24 Tashbayev B, Rusthen S, Young A, et al. Interdisciplinary, comprehensive oral and ocular evaluation of patients with primary Sjögren's syndrome. Sci Rep 2017;7:10761. https://doi.org/10.1038/ s41598-017-10809-w

25 Perricone C, Shoenfeld N, Agmon-Levin N, et al. Smell and autoimmunity: a comprehensive review. Clin Rev Allergy Immunol 2013;45:87-96. https://doi.org/10.1007/s12016-012-8343-x

26 Doherty PC. On the nose: shared themes for the sensory and immune self. Nat Immunol 2003;4:1043-1045. https://doi.org/10.1038/ ni1103-1043

27 Shoenfeld N, Agmon-Levin N, Flitman-Katzevman I, et al. The sense of smell in systemic lupus erythematosus. Arthritis Rheum 2009;60:1484-1487. https://doi.org/10.1002/art.24491

28 Bombini MF, Peres FA, Lapa AT, et al. Olfactory function in systemic lupus erythematosus and systemic sclerosis. A longitudinal study and review of the literature. Autoimmun Rev 2018;17:405-412. https:// doi.org/10.1016/j.autrev.2018.02.002

29 Takano K, Yamamoto M, Kondo A, et al. A clinical study of olfactory dysfunction in patients with Mikulicz's disease. Auris Nasus Larynx 2011;38:347-351. https://doi.org/10.1016/j.anl.2010.11.008 